\title{
Gradient Index Multimode Interference Structures - Technology and Applications
}

\author{
M. BŁAhut* AND D. KASPRZAK \\ Department of Optoelectronics, Silesian University of Technology \\ Krzywoustego 2, 44-100 Gliwice, Poland
}

\begin{abstract}
The aim of this paper is to present the operating principles and properties of gradient index multimode interference structures made by $\mathrm{K}^{+}-\mathrm{Na}^{+}$ion exchange in glass and their basic applications in optoelectronic circuits. The investigations of multimode interference structures were performed applying a method based on the visualization of light distribution in gradient structures, using fluorescence of the substance covering the multimode interference structures section. Basing on the above we present gradient index multimode interference structures applications in splitters and couplers $1 \times N$ technology of different configurations.
\end{abstract}

PACS numbers: 42.79.Gn, 42.82.Ds, 42.79.-e

\section{Introduction}

Multimode interference (MMI) structures have been for a few years subject to intensive research studies [1]. A large majority of works on MMI concerned interference structures made on the basis of step-index waveguides. In the year 2000, in the paper [2], the application of MMI in graded-index structures was proposed for the first time. Gradient structures made by ion exchange in glass are particularly attractive for MMI technology. Ion exchange technique making use of multi-step diffusion processes, electrodiffusion, heating, diffusive and electrodiffusive burying allows obtaining modal properties of the obtained waveguides which decide on intermode interference effects to be easily changed. In paper [3] we propose an experimental method which allows the visualization of effects of modal interference in MMI structures as well as some testing results for gradient index MMI structures made by $\mathrm{K}^{+}-\mathrm{Na}^{+}$and $\mathrm{Ag}^{+}-\mathrm{Na}^{+}$ion exchange in glass. The method uses fluorescence of the substance covering the MMI section. In this paper, using this method we show the experimental dependence of light propagation on the window width, technological process parameters of guided waves for structures made by $\mathrm{K}^{+}-\mathrm{Na}^{+}$ion exchange. Basing on the above we present gradient index MMI applications in splitters and couplers $1 \times N$ technology of different configurations and measurements of excess losses and power unbalance at the output of the splitters.

\section{Technology}

It will be useful at the very beginning to review briefly the basic properties of multimode interference structures [1]. The scheme of MMI structure is shown in Fig. 1.

\footnotetext{
* corresponding author; e-mail: marek.blahut@polsl.pl
}

It consists of a group of single-mode waveguides (a), which define the input field, of the wide multimode section (b) (typically monomode in $Y$ direction), where the interference effects of modal fields are observed and output single-mode waveguides (c).

(a)

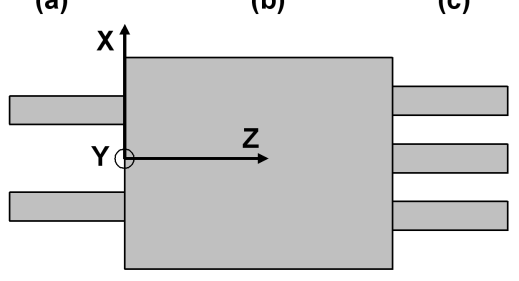

Fig. 1. The scheme of MMI structure.

Single-mode waveguides (a) provide stable distribution of the input field at the beginning of MMI. This field introduced to the interference section is decomposed into the modal fields of all modes of multimode waveguide. Each mode of the MMI section propagates with a different phase velocity and hence the field at the distance $z$ is a superposition of all modal fields with the same excitation coefficients and different phase shifts. As a result of it we observe the interference pattern which changes with the propagation length.

The investigated MMI sections are produced in the ion exchange process over $1 \mathrm{~h}$ and at temperature of $400^{\circ} \mathrm{C}$ in borosilicate glass. The diffusion is performed through the mask openings, in which, depending on the direction of excitation, symmetric or paired interference can be observed [2]. The widths $W_{\mathrm{M}}$ of the masks of MMI sections are 30,39, 51, 60, 69 and $81 \mu \mathrm{m}$.

Experimental results are compared with beam propagation method (BPM) simulation. The distribution profile of refractive index of MMI section obtained in the diffusion process is calculated numerically from the non- 
linear diffusion equation [4]:

$$
\frac{\partial C_{\mathrm{K}}}{\partial t}=\nabla\left[\frac{D_{\mathrm{K}}}{1-(1-m) C_{\mathrm{K}}} \nabla C_{\mathrm{K}}\right],
$$

where $C_{\mathrm{K}}$ is the dopant $\mathrm{K}^{+}$ions concentration, proportional to the refractive index change. Material parameters of the technological process - self-diffusion coefficient of $\mathrm{K}^{+}$ions $D_{\mathrm{K}}$, the mobility ratio $m$ of the ions $\mathrm{K}^{+}$and $\mathrm{Na}^{+}$, and the maximum of the refractive index change were determined by measurements of respective planar index profiles using inverse Wentzel, Kramers, Brillouin Method (IWKB) method. Figure 2 presents distribution profiles of the refractive index for the diffusion process of potassium ions at temperature $400^{\circ} \mathrm{C}$ and time $165 \mathrm{~h}$ obtained by IWKB method [4]. The determined self-diffusion coefficient is for both profiles equal to $2.18 \mu \mathrm{m}^{2} / \mathrm{h}$, whereas the distribution maxima on the surface are equal respectively to 0.0095 and 0.0117 . The observed differences in the distribution of the refractive index for both orthogonal polarizations result from the anisotropy of strains taking place during the technological process, and are of significant importance for performance characteristics of the MMI.

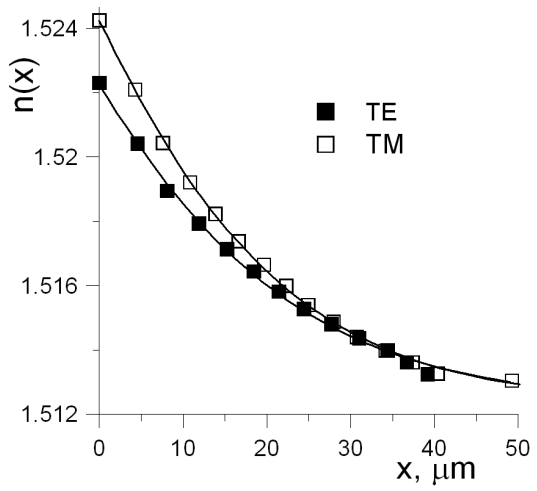

Fig. 2. Profiles of refractive index for TE and TM waves in BK-7 glass, - TM waves, $\square-\mathrm{TE}$ waves.

\section{Experimental method}

The investigations of MMI structures are carried out in the arrangement described in the paper [3], shown in Fig. 3. The MMI section, in which the mode field's interference is observed, excited from a laser by light of the wavelength $\lambda=0.63 \mu \mathrm{m}$, is covered with fluorescent substance by spin-coating. This substance is the Nile Blue "A" perchlorate suspension in PMMA. The fluorescent substance produces lightening proportional to the energy of the excited mode. The recording of the successive sequences of mode field interference patterns, by a CCD camera on $\mathrm{PC}$, makes it possible to find the propagation lengths for $N$-fold images.

Presented arrangement allows to investigate the MMI structures excited by the polarized (TE or TM) field from laser through the polarizer and unpolarized field from the

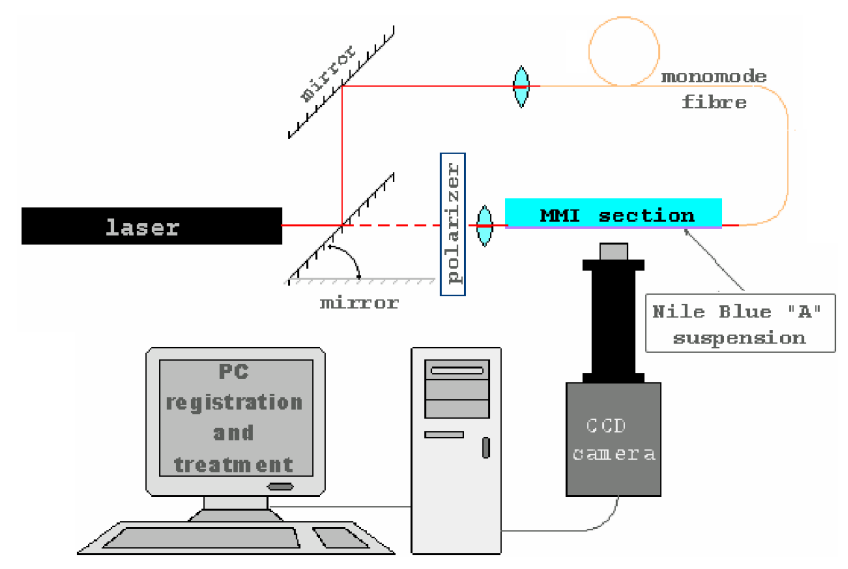

Fig. 3. Experimental arrangement.

single-mode fibre. That makes it possible to determine the dependence of propagation lengths for $N$-fold images on the window width, and the polarization of guided light.

Figure 4 presents, for example, successive sequences of the recorded mode field interference images for gradient index MMI section made by $\mathrm{K}^{+}-\mathrm{Na}^{+}$ion exchange for the window width $60 \mu \mathrm{m}$. The input field coming out from single-mode waveguide extends as a result of diffraction, reaching waveguide boundaries. Then, during successive reflections, the field matches to the mode structure and next the interference patterns evolution along the propagation length is observed. The section is symmetrically excited and the effects of symmetric interference are observed. Distinctly visible are $1 \times 6,1 \times 5$, $1 \times 4$ and $1 \times 3 \mathrm{~N}$-fold images marked in the interference pattern. The lengths of $N$-fold images depend on the polarization of guided light.

\section{Analysis of experimental results}

On the basis of the interference patterns analysis we determine the dependences which describe light propagation in MMI gradient structures. In Fig. 5 we present the dependences of the length of $N$-fold images on the window width for the symmetric interference. Experimental dependences are compatible with the equation for step-index waveguides which shows quadratic relation between the section width and self-imaging distance. Figure 6 shows the comparison of experimental results of $N$-fold images distances with BPM simulations for the MMI section of the window width $60 \mu \mathrm{m}$ for the symmetric interference. The propagation length for $N$-fold images decreases in comparison with numerical predictions together with the increase of the propagation distance. It can be observed that the effect is bigger for TM polarization and that the results for unpolarized light from the fiber are close to the results for TE polarization. The deviations can be probably explained by stresses. The participation of stresses in the refractive index formation is important - in BK-7 glass it is $30 \%$ for TE and 


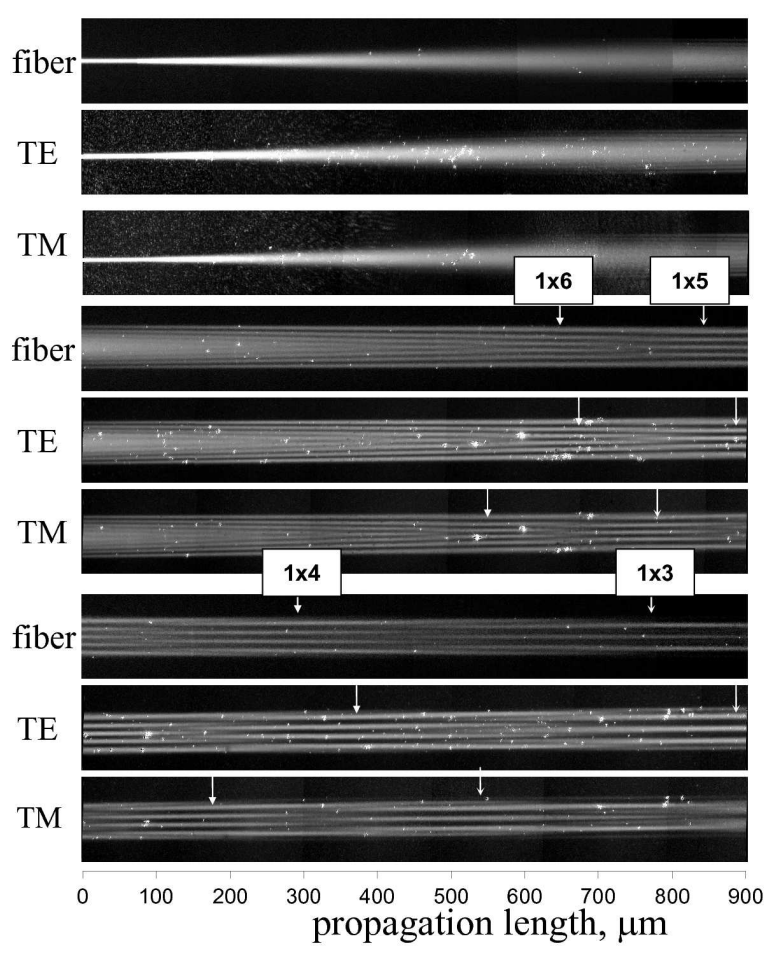

Fig. 4. Successive sequences of recorded mode field interference images for MMI section of the width $60 \mu \mathrm{m}$ symmetrically excited.

$40 \%$ for TM waves of the refractive index change [5-7]. Stresses depend on the time of the process and geometry of the waveguide. Therefore, the results obtained for strip waveguides produced over $1 \mathrm{~h}$ can differ from the numerical simulations based on the parameters obtained for the $1 \mathrm{D}$ process over $165 \mathrm{~h}$. It could be the reason of the different than quadratic dependence of propagation constants on mode number, which decides on self-imaging phenomena.

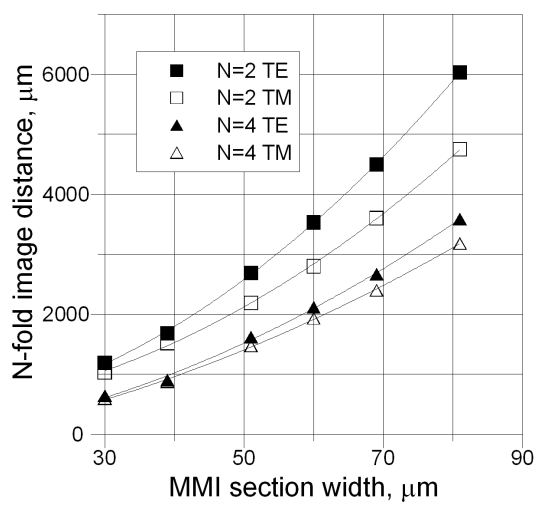

Fig. 5. The dependence of length of $N$-fold images on the window width for MMI section.

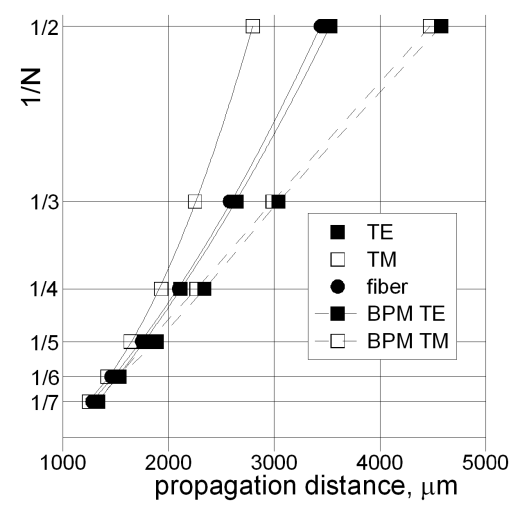

Fig. 6. The comparison between experimental and numerical values of $N$-fold images lengths; MMI section width is $60 \mu \mathrm{m}$.

\section{Gradient index MMI structure applications in couplers/splitters technology}

On the basis of self-imaging effects analysis, using potassium-sodium ion exchange we have designed and fabricated the $1 \times N$ waveguide splitters in symmetrical configuration. It should be underlined, however, that when designing splitters and couplers made by $\mathrm{K}^{+}-\mathrm{Na}^{+}$ ion exchange technology we cannot use analytical expressions from the theory for step-index waveguides. The geometry of each designed splitter and coupler has to be determined separately. The geometrical parameters of masks for the splitters $1 \times 2,1 \times 3,1 \times 4,1 \times 5$ are listed in Table I.

TABLE I

Geometrical parameters of MMI splitters.

\begin{tabular}{c|c|c|c}
\hline \hline $\begin{array}{c}\text { MMI section } \\
\text { width }[\mu \mathrm{m}]\end{array}$ & $1 \times N$ & $\begin{array}{c}\text { MMI section } \\
\text { length }[\mu \mathrm{m}]\end{array}$ & $\begin{array}{c}\text { Outputs } \\
\text { distance }[\mu \mathrm{m}]\end{array}$ \\
\hline 30 & $1 \times 2$ & 1120 & 14.5 \\
\hline 39 & $1 \times 2$ & 1630 & 15 \\
\hline \multirow{2}{*}{51} & $1 \times 2$ & 2600 & 19 \\
\hline 60 & $1 \times 3$ & 1980 & 14 \\
\hline 69 & $1 \times 4$ & 1670 & 16 \\
\hline \multirow{2}{*}{81} & $1 \times 4$ & 2720 & 14.5 \\
\hline & $1 \times 5$ & 3650 & 17.5 \\
& & & 14.5
\end{tabular}

Figure 7 shows the configuration of an experimental stand for determining the parameters of MMI splitters. The input signal coming out from the input single-mode waveguide is divided in $Y$ junction to the MMI splitter and the single-mode waveguide which works as a reference arm. The signals at the output of MMI splitter are compared to the signal from the reference arm. That enables us to measure the excess losses and the signals unbalances at the output. 
Gradient index splitters parameters.

\begin{tabular}{c|c|c|c|c|c|c|c|c}
\hline \hline $\begin{array}{c}\text { Splitter } \\
1 \times N, \\
\text { width }\end{array}$ & $\alpha[\mathrm{dB}]$ & Unbalance & $A_{1}$ & $A_{2}$ & $A_{3}$ & $A_{4}$ & $A_{5}$ & $A_{0}$ \\
\hline $\begin{array}{c}1 \times 2 \\
30 \mu \mathrm{m}\end{array}$ & 1.4 & $3 \%$ & 0.58 & 0.61 & - & - & - & 1 \\
$1 \times 3$ & & & & & & & & \\
$51 \mu \mathrm{m}$ & 3.9 & $2 \%$ & 0.36 & 0.36 & 0.38 & - & - & 1 \\
$1 \times 4$ & & & & & & & & \\
$69 \mu \mathrm{m}$ & 5.9 & $3-8 \%$ & 0.25 & 0.22 & 0.22 & 0.31 & - & 1 \\
$1 \times 5$ & & & & & & & & 1 \\
$81 \mu \mathrm{m}$ & 10.5 & $1-6 \%$ & 0.17 & 0.11 & 0.11 & 0.12 & 0.14 & 1
\end{tabular}

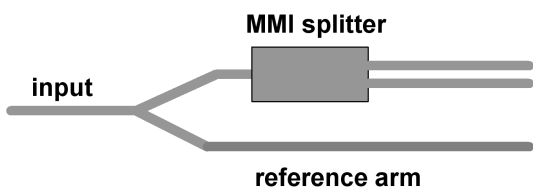

Fig. 7. Configuration of experimental stand for measurements of excess loss and signal unbalance.

The registration of output signals was performed in the visualization stand presented in Fig. 3. The fluorescence substance covered the output ports and a part of the reference arm. Figures $8 \mathrm{a}-\mathrm{c}$ show the power distributions at the outputs which enables to determine the excess losses and power unbalances of the outputs fields. In Table II we present the measured excess losses, un-
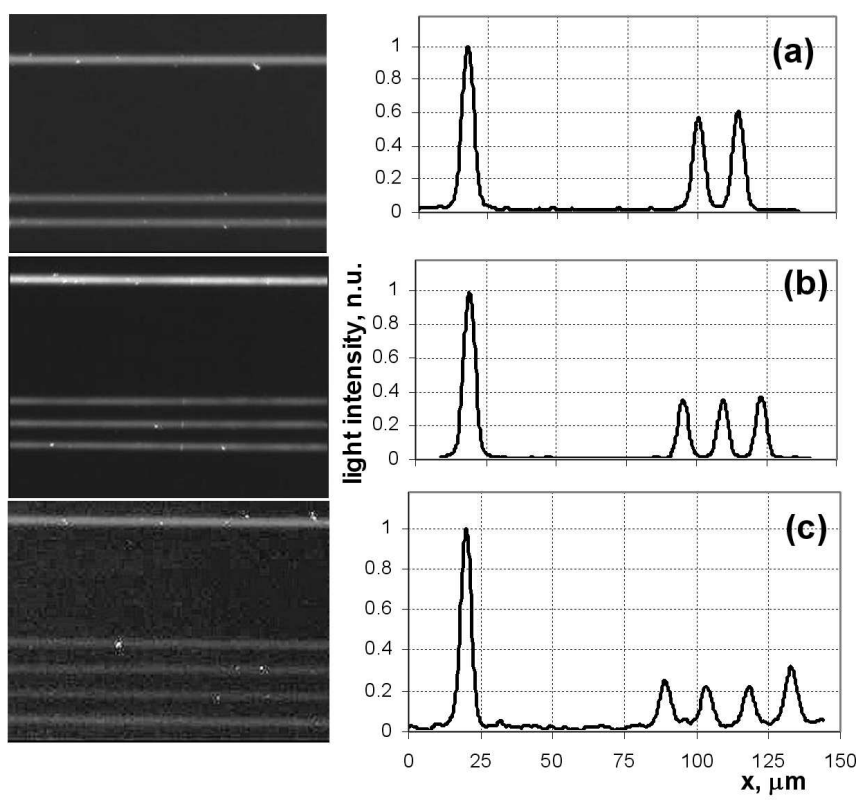

Fig. 8. The visualization (on left) and linear profile (on right) of output signals in symmetrical splitters $1 \times N$ of the window width $W$ of MMI section: (a) $1 \times 2, W=$ $30 \mu \mathrm{m}$; (b) $1 \times 3, W=51 \mu \mathrm{m}$; (c) $1 \times 3, W=69 \mu \mathrm{m}$. balances and amplitude distribution at the output of the selected splitters. Excess losses increase with the number of output ports and the MMI section width.

\section{Conclusion}

The paper presents experimental investigations of MMI structures produced by $\mathrm{K}^{+}-\mathrm{Na}^{+}$ion-exchange. The investigations concern self-imaging phenomena of the input field for symmetrical interference depending on the polarization of guided light. It can be confirmed that the propagation length for the $N$-fold image for symmetric interference depends on the MMI section width similarly as in the theory for step-index waveguides. The propagation length for $N$-fold images decreases in comparison with numerical predictions together with the increase of the propagation distance. These effects result probably from stresses which are important for $\mathrm{K}^{+}-\mathrm{Na}^{+}$ ion exchange. On the basis of the self-imaging effects analysis we have designed and fabricated the $1 \times N$ waveguide splitters in symmetrical configuration. We propose an experimental stand for the measurements of the excess losses and power unbalance at the output. We have reported that the excess losses increase with the number of output ports and the MMI section width.

\section{References}

[1] L.B. Soldano, E.C.M. Pennings, J. Lightwave Technol. LT-13, 615 (1995).

[2] S. Das, D. Geraghty, S. Honkanen, N. Peyghambarian, Proc. SPIE 3936, 239 (2000).

[3] M. Błahut, P. Karasiński, D. Kasprzak, R. Rogoziński, Opt. Commun. 214, 47 (2002).

[4] M. Błahut, A. Opilski, R. Rogoziński, Opt. Appl. 22, 161 (1992).

[5] D. Dorosz, K. Barczak, T. Pustelny, J. Dorosz, Acta Phys. Pol. A 114, A-61 (2008).

[6] T. Pustelny, E. Maciak, Z. Opilski, M. Bednorz, Opt. Appl. 37, 187 (2007).

[7] A.A. Brandenburg, J. Lightwave Technol. LT-4, 1580 (1986). 